\title{
Effects of mass transfer on a resonance instability in the laminar boundary layer flow over a rotating-disk
}

\author{
M TURKYILMAZOGLU \\ Mathematics Department, University of Hacettepe, 06532-Beytepe, Ankara, \\ Turkey \\ e-mail: turkyilm@hotmail.com
}

MS received 17 September 2008; revised 24 March 2009

\begin{abstract}
Direct spatial resonance phenomenon occurring in the viscous incompressible boundary layer flow due to a rotating-disk is investigated in this paper based on the linear stability theory. The possible effects of suction and injection are explored on the direct spatial resonance instability mechanism detected earlier in the case of zero-suction. This instability leads to an algebraic growth of disturbances while the flow is yet in the laminar regime and this in turn, may initiate the non-linearity and transition, competing with the unboundedly growing timeamplified perturbations. In line with the physical intuition, results show that suction delays the onset of resonance instability by increasing the critical Reynolds number, whereas it is enhanced by the presence of injection. The critical parameter for direct spatial resonance instability always precedes the onset value for absolute instability mechanism, after a comparison with the previous work. Therefore, in the case of suction, the onset parameter is close to the transition value as determined from the earlier experimental observations. It is further examined the inviscid nature of both absolute as well as direct spatial resonance instabilities when suction or injection is applied through the disk, and is demonstrated that these instability mechanisms are not in any way an artifact of the parallel flow approximation assumed during the linearization of viscous incompressible stability equations.
\end{abstract}

Keywords. Rotating-disk; viscous/inviscid linear stability; direct spatial resonance; suction or injection.

\section{Introduction}

Instability mechanisms leading to an unavoidable breakdown of laminar boundary layer and a consequent transition to turbulence have been the focus of substantial research in hydrodynamic stability problems of practical significance, in particular applications over sweptback wings. Recent advances point that in fully three-dimensional flows the main instability mechanism manifests itself in the form of crossflow vortices generated by viscous/inviscid stationary/non-stationary perturbations penetrated into the boundary layer and owing to the inflectional character of the mean flow. This instability mechanism and its subsequent causes 
may be better understood using a prototype three-dimensional rotating-disk boundary layer flow since the exact solution to the steady incompressible flow has been known since pioneering work of Kármán (1921). Within this connection, many theoretical and numerical works besides the experiments have been performed, and still going on, on the aspects of the stability of the rotating-disk flow to shed light on the crossflow vortex-induced instabilities. In the present study, a consequence of the crossflow instability, namely the direct spatial resonance event taking place in the three-dimensional rotating-disk boundary layer flow will be mainly investigated especially in the presence of mass transfer through the surface of the disk.

Perturbations introduced into the boundary layer and exposed to the crossflow instability may undergo two types of evolutions, convectively or absolutely. The convective or absolute instability nature of the instability can be extracted from the singularities arising in the dispersion relationship obtained as a result of linearizing the governing equations of motion in the vicinity of the basic steady Von Karman's flow. The singular points form whenever modes associated with waves propagating in opposite or same directions merge. If the coalescing branches originate from the waves propagating in opposite directions, the singularity which causes resonance is said to be of pinch type, which separates an absolutely unstable region from a convectively unstable one. If such a pinching occurs in an unstable system then the response of the flow to a localized line forcing will reveal time-amplified disturbances growing both upstream and downstream of the point of excitation. If, on the other hand, the two coalescing modes originate from waves propagating in the same direction, then the corresponding singularity is of an ordinary branch point, called double-pole type. In the existence of such a singularity, the amplified disturbances will at the same time convect away from the source of excitation leaving eventually the flow undisturbed.

Until 1995, almost all research was intensively concentrated on the convective type of instability, the second scenario as described above. For example, Gregory et al (1955), Wilkinson \& Malik (1983), Kohama et al (1987), Mack (1985), Malik (1986), Hall (1986), Bassom \& Gajjar (1988), Balakumar \& Malik (1990) and Balachandar et al (1992), amongst many others highlighted that primary and secondary instabilities of stationary/travelling viscous/inviscid perturbations amplify convectively to give rise to the transition to turbulence. In line with the first scenario, Lingwood (1995) discovered pinching phenomenon for the coalescing waves emerging from distinct halves of the complex wavenumber plane before coalescing. Thus, Lingwood (1995) showed that the Von Karman's boundary layer flow becomes radially absolutely unstable (the circumferential symmetry allowed this to hold), i.e. the response to a transient disturbance grows with time at fixed radial positions above a critical Reynolds number of about 507. The existence of absolute instability was later verified in the experiment of Lingwood (1996), in which it was suggested that the absolute instability mechanism encountered is responsible for the onset of non-linear behaviour and laminar-turbulent transition, of course in the absence of possible more dominant mechanisms. Following the analysis of Lingwood (1995) together with the Briggs-Bers pinching requirement, see for instance Briggs (1964), Bers (1975), Huerre \& Monkewitz (1990) and Turkyilmazoglu (2003), Turkyilmazoglu et al (2000) showed that compressible flow over a rotating-disk is also inviscidly absolutely unstable over a numerically determined specific parameter range.

To stabilize the aforementioned instabilities operating in three-dimensional flows either wall-compliance or suction/blowing techniques are generally made use of in practical flows, at least to delay the onset of transition. Cooper \& Carpenter (1997a,b) for instance showed substantial stabilizing effects on the upper branch crossflow instability as well as on the absolute instability by pushing the critical Reynolds number to as higher values as desired. They further concluded that beyond a critical level of wall compliance the complete suppression of 
the absolute instability is possible, removing a major route to transition in the Von Karman's boundary layer flow. Likewise to the wall compliance, suction is generally applied to maintain laminar flow through decreasing the thickness of the boundary layer as well as reducing the magnitude of the crossflow velocity, see for example the attachment-line work of Hall et al (1984) and the linear stability work of Dhanak (1992) for the rotating-disk flow. In Dhanak (1992) it was shown that suction increases the critical Reynolds number of the convective instability regime whereas injection has a reverse effect, conversely destabilizing the flow over a rotating-disk. By extending the non-linear triple-deck analysis of Mackerrel (1987), Bassom \& Seddougui (1992) further investigated the influence of suction/injection on the non-linear stability of stationary lower branch crossflow modes at asymptotically large Reynolds number and later this analysis was generalized to compressible flow over a rotatingdisk in Seddougui \& Bassom (1996). They found in both investigations that suction lowers the threshold amplitude for the initial growth of disturbances, whereas injection raises it. Thus, even though from a linear viewpoint suction is stabilizing as far as the convective instability is concerned, see Dhanak (1992), non-linearity has a destabilizing impact for suction since the threshold amplitude for the decaying disturbances decreases with increasing suction. Contrary to the suction case, non-linearity has stabilizing influence on the fluid injection. Based on this finding, Bassom \& Seddougui (1992) and Seddougui \& Bassom (1996) suggested that an experiment with fluid injection would need strong forcing for subcritical instability to take place and less forcing with suction. The compressibility effects on the suction/blowing were also found to be destabilizing in Seddougui \& Bassom (1996). Although the results of Seddougui (1990) indicated that an unstable mode of instability is more likely to occur for a compressible flow with highly cooled wall than for an incompressible flow, the results of Seddougui \& Bassom (1996) showed that for a highly cooled wall the imposition of blowing decreases the likelihood of non-linear effects causing the early breakdown of the laminar flow.

The above research is so far restricted to the analysis of suction/blowing effects on the convective nature of instabilities. The effects of mass transfer through the disk on the absolute instability mechanism were later assessed in Lingwood (1997) within the scope of linear stability theory. Uniform suction through the disk was demonstrated to delay the onset of absolute instability, while uniform injection promotes the onset as for the convective instability case.

The current work is devoted to the effects of mass transfer through the disk surface on the direct spatial resonance instability mechanism, already shown to exist without the mass transfer in incompressible flow in Turkyilmazoglu \& Gajjar (2000). A spatial resonance instability mechanism appears in the second scenario as outlined previously, where a doublepole singularity takes place when the merging modes originating in the same wavenumber planes are nearly neutral, an asymptotic analysis reveals that, the damping rates are very small causing a short-term algebraic growth for small times or short distances. Such a mechanism may compete before the exponentially growing modes and first trigger the non-linearity in the boundary layers followed by transition. Benney \& Gustavsson (1981) for instance investigated the direct spatial resonance in parallel shear flows between an Orr-Sommerfeld and a Squire mode. Koch (1986) and Shanthini (1989) also carried out such an investigation in their numerical study of the stability of Blasius boundary layer flow on a flat plate and planePoiseiulle flow. The recent study of Turkyilmazoglu \& Gajjar (2000) showed that algebraic growth of the perturbations through the direct spatial resonance is a natural phenomenon taking place while the incompressible Von Karman's boundary layer flow is still in the laminar state. It was also conjectured in Turkyilmazoglu \& Gajjar (2000) that incorporating the non-parallel 
terms into the stability analysis might influence the absolute instability character of the flow by shifting the critical Reynolds number for the onset of absolute instability to a turbulent regime, and that the onset of direct spatial resonance will still persist to occur below the critical Reynolds number for the underlying flow to be transitional. This has been indeed verified in the non-parallel numerical simulation results of Davies \& Carpenter (2003), in which no absolute instability was detected for the critical value found in Lingwood (1995). As a result, the absolute instability route to transition gets weakened and hence transition is most probably driven through the direct spatial resonance instability mechanism. Moreover, the recent study of Koch (2002) on the swept-wing boundary layer on the spatio-temporal stability of primary and secondary crossflow vortices failed to detect any absolute instability but as concluded, instead the transition is most probably occurred through the convective instability as in the experiments carried out on DLR swept-plate.

Motivated by the above arguments, we here seek for the critical Reynolds number and parameter domain leading to direct spatial resonance, which is relevant to the convective instability mechanism, taking into consideration uniform suction or injection through the disk. Therefore, the governing equations of the rotating-disk boundary layer flow are linearized by imposing upon small disturbances, and a self-consistent asymptotic approach introduced in Turkyilmazoglu \& Gajjar (2000) is undertaken, unlike the conventional parallel flow assumption. When non-parallel terms are ignored with an additional assumption of $r$ (the radial distance from the center of the disk) being set to unity, the traditional sixth-order stability equations are obtained. These equations, which have been treated before by Malik (1986) and Balakumar \& Malik (1990), are solved here using a different technique based on Spectral Chebyshev collocation with a staggered grid structure. A Runge-Kutta scheme was also employed for checking the self-consistency of our results. The use of spatio-temporal linear stability analysis shows that three families of eigenfunctions, having positive or negative energies, are possible in the rotating-disk flow, and branch interchanging between distinct waves points to the existence of resonance instability mechanisms. We initially examine the linear absolute instability of the inviscid modes when suction is under consideration and show that suction has a greatly stabilizing effect as opposed to the destabilizing influence of blowing. Next, the direct spatial resonance of the viscous flow is investigated followed by the inviscid analysis. The match of the viscous instability modes, as found here in the infinite Reynolds number limit to the inviscid modes strongly suggests that the resonance instability identified here cannot be an artifact of the parallel-flow approximation employed during the linearization of the stability equations.

This paper is organized in the following manner. The governing equations and the mean flow are in given $\S 2$, followed by the derivation of the linear viscous and inviscid stability equations in $\S 3$. The numerical technique to discretize and solve the stability equations is briefly described in $\S 4$. They direct spatial resonance results for the viscous modes as well as the absolute instability results for the inviscid modes are presented in $§ 5$. Conclusions are provided in $\S 6$.

\section{Formulation of the problem}

\subsection{Governing equations of the flow}

We consider the three-dimensional boundary-layer flow of an incompressible fluid on an infinite disk which rotates about its axis with a constant angular velocity $\Omega$. The NavierStokes equations are non-dimensionalized with respect to a length scale $L=r_{e}^{*}$, velocity 
scale $U_{c}=L \Omega$, time scale $L / U_{c}$ and pressure scale $\rho U_{c}^{2}$, where $\rho$ is the fluid density. This leads to a global Reynolds number $\operatorname{Re}=\frac{U_{c} L}{v}=R^{2}$, where $R$ is the Reynolds number based on the displacement thickness $\delta=\left(\frac{v}{\Omega}\right)^{\frac{1}{2}}$. Thus relative to non-dimensional cylindrical polar coordinates $(r, \theta, z)$ which rotate with the disk, the full time-dependent, unsteady NavierStokes equations governing the viscous fluid flow are the usual momentum and the continuity equations, which are given by

$$
\begin{aligned}
& \frac{\partial u}{\partial t}+u \frac{\partial u}{\partial r}+\frac{v}{r} \frac{\partial u}{\partial \theta}+w \frac{\partial u}{\partial z}-\frac{v^{2}}{r}-2 v-r=-\frac{\partial p}{\partial r}+\frac{1}{R^{2}}\left[\nabla^{2} u-\frac{2}{r^{2}} \frac{\partial v}{\partial \theta}-\frac{u}{r^{2}}\right], \\
& \frac{\partial v}{\partial t}+u \frac{\partial v}{\partial r}+\frac{v}{r} \frac{\partial v}{\partial \theta}+w \frac{\partial v}{\partial z}+\frac{u v}{r}+2 u=-\frac{1}{r} \frac{\partial p}{\partial \theta}+\frac{1}{R^{2}}\left[\nabla^{2} v+\frac{2}{r^{2}} \frac{\partial u}{\partial \theta}-\frac{v}{r^{2}}\right], \\
& \frac{\partial w}{\partial t}+u \frac{\partial w}{\partial r}+\frac{v}{r} \frac{\partial w}{\partial \theta}+w \frac{\partial w}{\partial z}=-\frac{\partial p}{\partial z}+\frac{1}{R^{2}}\left[\nabla^{2} w\right], \\
& \frac{\partial u}{\partial r}+\frac{1}{r} \frac{\partial v}{\partial \theta}+\frac{\partial w}{\partial z}+\frac{u}{r}=0 .
\end{aligned}
$$

In this analysis the fluid is assumed to lie in the $z \geq 0$ semi-infinite space. Equations (1) contain the viscous, streamline curvature effects as well as the effects stemming from the Coriolis forces. It is now well-known that these terms have a strongly stabilizing impact in the linear stability theory, as pointed out by Malik \& Poll (1985), Wilkinson \& Malik (1985).

\subsection{Mean flow}

The dimensionless mean flow velocities and pressure are given by Von Karmán's exact selfsimilar solution of the Navier-Stokes equations for steady laminar flow in the case of zerosuction. Since the wall normal velocity is constant when the suction or injection is considered, the Von Karmán's similarity solution will still be valid except with the appearance of a uniform upwards or downwards flow through the surface of the disk. Hence, the boundary-layer coordinate $Z$, which is of order $O(1)$ is defined as $Z=z R$, and the self-similar equations take the form

$$
\left(u_{B}, v_{B}, w_{B}, p_{B}\right)=\left(r U[Z], r V[Z], \frac{1}{R} W[Z], \frac{1}{R^{2}} P[Z]\right),
$$

where the functions $U, V, W$ and $P$ satisfy the following ordinary differential equations

$$
\begin{aligned}
& U^{2}-(V+1)^{2}+U^{\prime} W-U^{\prime \prime}=0, \\
& 2 U(V+1)+V^{\prime} W-V^{\prime \prime}=0, \\
& P^{\prime}+W^{\prime} W-W^{\prime \prime}=0, \\
& 2 U+W^{\prime}=0 .
\end{aligned}
$$

Here, primes denote derivative with respect to $Z$ and the appropriate boundary conditions are given as

$$
\begin{aligned}
& U=W=W+s=0 \text { at } Z=0, \\
& U=0, V=-1, W=h_{\text {inf }} \text { as } Z \rightarrow \infty .
\end{aligned}
$$


The unknown $h_{\text {inf }}$ is a constant vertical velocity of the rotating fluid in the far-field above the disk, and its value has to be found numerically in the course of the solution of equations (3) and (4). Moreover, $s$ is a positive constant for suction through the disk and is negative constant for blowing.

\section{Linear stability equations}

\subsection{Viscous disturbance equations}

We are here interested in perturbation solutions of Von Kármán's self-similarity velocity profiles (2). The instantaneous non-dimensionalized velocity components imposed on the basic steady flow are $u, v, w$ and the pressure component is $p$ and they can be expressed as

$$
(u, v, w, p)=\left(u_{B}, v_{B}, w_{B}, p_{B}\right)+\left(u^{\prime}, v^{\prime}, w^{\prime}, p^{\prime}\right) .
$$

Infinitesimally small disturbances $u^{\prime}, v^{\prime}, w^{\prime}$ and $p^{\prime}$ are superimposed on the steady flow obtained from the solution of (3-4). The disturbance components of the above system are later determined by solving the form of the Navier-Stokes equations that result from substituting these quantities into (1), and subtracting out the mean flow equations, satisfying (3). Having linearized the equations for small perturbations, we find that the linearized Navier-Stokes operator has coefficients independent of $\theta$ and hence the disturbances can be decomposed into a normal mode form proportional to $e^{i R(\beta \theta-\bar{\omega} t)}$. Such an approximation leads the disturbances to be wave-like, separable in $\theta$ and $t$. Consequently, the perturbations may be assumed to be of the form

$$
\left(u^{\prime}, v^{\prime}, w^{\prime}, p^{\prime}\right)=(\tilde{u}[r, Z], \tilde{v}[r, Z], \tilde{w}[r, Z], \tilde{p}[r, Z]) e^{i R(\beta \theta-\bar{\omega} t)}+c . c .,
$$

where $\beta$ and $\bar{\omega}$ respectively are the wave number in the azimuthal direction and the scaled frequency of the wave propagating in the disturbance wave direction.

The separation in $\theta$ and $t$ simplifies the linear system of equations. However no such simplification arises as far as the $r$-dependence is concerned (except in the limit as $R \rightarrow \infty$ ) and the full linearized partial differential system has to be solved subject to suitable initial conditions to determine the stability of the flow. Consider next the limit $R \rightarrow \infty$ and introduce the scale $X=R r$ which is the appropriate scale on which the disturbances develop. After allowing for the multiple-scale replacement of $\frac{\partial}{\partial r}$ by

$$
R \frac{\partial}{\partial X}+\frac{\partial}{\partial r}
$$

and keeping only terms of up to $O(1 / R)$ the following linear system is obtained

$$
\begin{aligned}
& -i \bar{\omega} \tilde{u}+r U \frac{\partial \tilde{u}}{\partial X}+i \beta V \tilde{u}+r \frac{d U}{d Z} \tilde{w}+\frac{\partial \tilde{p}}{\partial X} \\
& =-\frac{1}{R}\left[W \frac{\partial \tilde{u}}{\partial Z}+U \tilde{u}-2(V+1) \tilde{v}-\nabla_{2}^{2} \tilde{u}\right]-\frac{1}{R}\left[r U \frac{\partial \tilde{u}}{\partial r}+\frac{\partial \tilde{p}}{\partial r}\right], \\
& -i \bar{\omega} \tilde{v}+r U \frac{\partial \tilde{v}}{\partial X}+i \beta V \tilde{v}+r \frac{d V}{d Z} \tilde{w}+i \frac{\beta}{r} \tilde{p} \\
& =-\frac{1}{R}\left[W \frac{\partial \tilde{v}}{\partial Z}+U \tilde{v}+2(V+1) \tilde{u}-\nabla_{2}^{2} \tilde{v}\right]-\frac{1}{R}\left[r U \frac{\partial \tilde{v}}{\partial r}\right],
\end{aligned}
$$




$$
\begin{aligned}
& -i \bar{\omega} \tilde{w}+r U \frac{\partial \tilde{w}}{\partial X}+i \beta V \tilde{w}+\frac{\partial \tilde{p}}{\partial Z}+\frac{1}{R}\left[W \frac{\partial \tilde{w}}{\partial Z}+\frac{d W}{d Z} \tilde{w}-\nabla_{2}^{2} \tilde{w}\right] \\
& =-\frac{1}{R}\left[r U \frac{\partial \tilde{w}}{\partial r}\right], \\
& \frac{\partial \tilde{u}}{\partial X}+\frac{1}{R r} \tilde{u}+\frac{i \beta}{r} \tilde{v}+\frac{\partial \tilde{w}}{\partial Z}=-\frac{1}{R} \frac{\partial \tilde{u}}{\partial r} .
\end{aligned}
$$

The operator $\nabla_{2}^{2}$ is defined by

$$
\nabla_{2}^{2}=\frac{\partial^{2}}{\partial X^{2}}+\frac{\partial^{2}}{\partial Z^{2}}-\frac{\beta^{2}}{r^{2}}
$$

The terms on the right hand side of (5) reflect the non-parallelism of the basic flow and appear at the same order as the other $O(1 / R)$ terms which are retained in the familiar 'parallel flow approximation'. In the formal limit $R \rightarrow \infty$ and with $\frac{\partial}{\partial X}$ replaced by $i \alpha$ we obtain Rayleigh's equation. It is only in this limit that the full normal mode decomposition can be justified. If we neglect the terms on the right hand side of (5) and replace $\frac{\partial}{\partial X}$ by $i \alpha$ together with $r=1$, the sixth-order system studied by several people including Mack (1984), Malik (1986), Balakumar \& Malik (1990) and Lingwood (1995), amongst others, is retrieved. Setting $r=1$ as highlighted above is tantamount to considering the stability at the local station $r_{e}^{*}$.

The reduced system of equations stemming from these approximations can be written in the following form

$$
\begin{aligned}
& \tilde{u}^{\prime \prime}-W \tilde{u}^{\prime}-\left[i R(\alpha U+\beta V-\bar{\omega})+\lambda^{2}+U\right] \tilde{u}+2(V+1) \tilde{v}-R U^{\prime} \tilde{w}-i \alpha R \tilde{p}=0, \\
& \tilde{v}^{\prime \prime}-W \tilde{v}^{\prime}-\left[i R(\alpha U+\beta V-\bar{\omega})+\lambda^{2}+U\right] \tilde{v}-2(V+1) \tilde{u}-R V^{\prime} \tilde{w}-i \beta R \tilde{p}=0, \\
& \tilde{w}^{\prime \prime}-W \tilde{w}^{\prime}-\left[i R(\alpha U+\beta V-\bar{\omega})+\lambda^{2}+W^{\prime}\right] \tilde{w}-R \tilde{p}^{\prime}=0, \\
& \bar{\alpha} \tilde{u}+i \beta \tilde{v}+\tilde{w}^{\prime}=0,
\end{aligned}
$$

where $\lambda^{2}=\alpha^{2}+\beta^{2}, \bar{\alpha}=i \alpha+\frac{1}{R}$ and $\omega=\bar{\omega} R$.

The boundary conditions for this set of equations are $\tilde{u}=\tilde{v}=\tilde{w}=0$ at the solid wall $(Z=0)$. Considering the decaying property of disturbances the boundary conditions to be imposed far away from the disk surface are derived from the asymptotic form of equations (6). The specific form of the far-field boundary conditions can be found in Turkyilmazoglu (1998).

\subsection{Inviscid Rayleigh equation}

It is possible to obtain the familiar Orr-Sommerfeld equation for the normal velocity component from the set of equations (6), by ignoring the streamline curvature and Coriolis effects. Further neglect of all the terms of order of $R^{-1}$ in (6) leads to the well-known Rayleigh equation

$$
\left[(\alpha U+\beta V-\omega)\left(D^{2}-\lambda^{2}\right)-\left(\alpha D^{2} U+\beta D^{2} V\right)\right] \tilde{w}=0,
$$

where $\lambda^{2}=\alpha^{2}+\beta^{2}, \omega=\bar{\omega}$ and $D=\frac{\partial}{\partial Z}$.

The homogeneous boundary conditions to be incorporated are given as

$$
\tilde{w}=0 \text { at } Z=0, \quad \tilde{w} \rightarrow 0 \text { as } Z \rightarrow \infty .
$$




\section{Results and Discussion}

In this section before presenting the results, a brief outline of the solution method that is used for the basic flow (3-4) and stability equations (6-7) is given. Basically, the mean flow equations were solved making use of a standard double precision fourth-order Runge-Kutta integrator in combination with a shooting applied at the infinity to determine the wall derivatives of the flow variables. In addition to a Runge-Kutta integrator, a Spectral Chebyshev collocation technique was employed to deal with the linear stability equations. In Spectral method, the semi-infinite physical domain was first mapped onto $[-1,1]$ finite interval via the transformation $\eta=-1+2 Z / Z_{\max }$ so that the momentum equations in (6) were collocated at Gauss-Lobatto points $\cos \left[k \frac{\pi}{N}\right]$, while the continuity equation was imposed at the Gauss points $\cos \left[\left(k+\frac{1}{2}\right) \frac{\pi}{N}\right]$. Here, $Z_{\text {max }}$ is the far-field boundary of the flow and $N$ denotes the number of collocation points. As a result of such a collocation, the imposition of the unknown pressure perturbation on the wall was avoided. With the help of a Chebyshev interpolation between the Gauss and Gauss-Lobatto points, see for instance Malik et al (1984), equations (6-7) were reduced to a matrix form $L \chi=B$, where $\chi=[\tilde{u}, \tilde{v}, \tilde{w}, \tilde{p}]^{\mathrm{T}}$ is the sought eigenvector and the coefficient matrix is associated with the eigenvalues $(\alpha, \beta, \omega, R)$. A solution technique for the eigenvalue problem as well as two different procedures to compute the branch points making use of Newton-Raphson method can be found in Turkyilmazoglu (1998).

\subsection{Mean flow results with suction and injection}

We present here the mean flow quantities $(U, V, W)$ with suction and injection included. Figures 1a-c show these quantities plotted against $\eta$ for a variety of parameters $s$. These figures reveals an excellent agreement with the basic flow profiles of Lingwood (1997) and Dhanak (1992). As computed during the integration of equations (3-4), figure 1d shows the values of $U^{\prime}(0), V^{\prime}(0)$ and $h_{\text {inf }}$, where prime corresponds to derivative with respect to the physical boundary layer coordinate $Z$. It can be inferred from these figures that the crossflow velocity profiles are inflectional in the radial directions and the inflexion points (indicated by the cross marks) are moving away from the wall as the injection is active while a reverse effect is seen when the suction is present. This is in consistence with the thinning effect of the boundary layer by decreasing the magnitude of radial velocity and thus reducing the three-dimensionality of the boundary layer as also outlined in Lingwood (1997) and Dhanak (1992). A characteristic feature of the parameters displayed in figure $1 \mathrm{~d}$ is that $V^{\prime}(0)$ and $h_{\text {inf }}$ vary monotonically with suction parameter $s$, whereas $U^{\prime}(0)$ does not, as also manifested from the large asymptotic values, see for instance Turkyilmazoglu (2005). Unlike to the suction, injection has an influence of increasing the magnitude of the radial velocity and also increasing the axial flow both near the rigid disk and far away from the disk. The effects of these basic velocities under the influence of suction and injection were previously investigated by Dhanak (1992) on the convective instability properties and by Lingwood (1997) on the absolute instability mechanism for the incompressible rotating-disk boundary layer flow. In what follows such effects will be explored on the existence of direct spatial resonance feature as also found in Turkyilmazoglu \& Gajjar (2000) with zero-suction case.

\subsection{Resonance results}

In order to verify the stability solver, we first demonstrated the convective neutral stability curves for a travelling wave with frequency $\omega=7.9$ in figure 2 , for the specified parameters $s=-0 \cdot 5,0$ and $0 \cdot 5$, respectively. This figure is essentially the same as the corresponding 


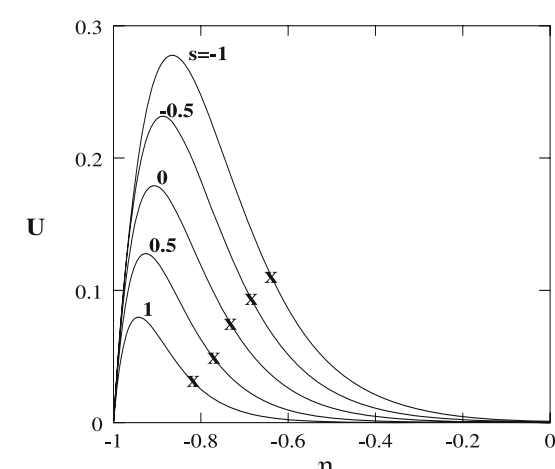

(a)

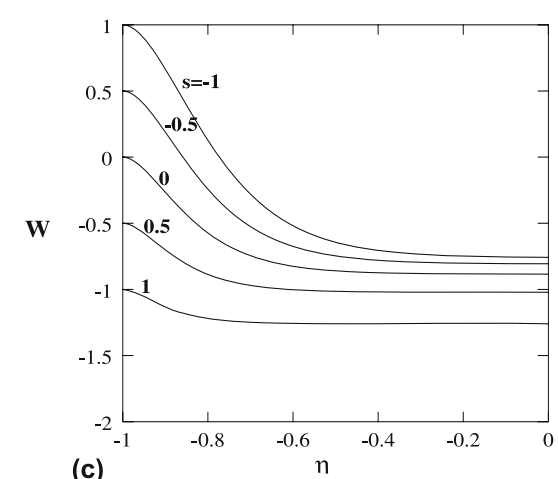

(c)

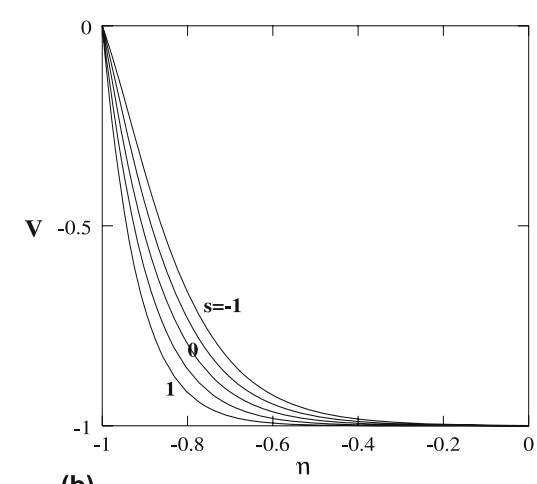

(b)

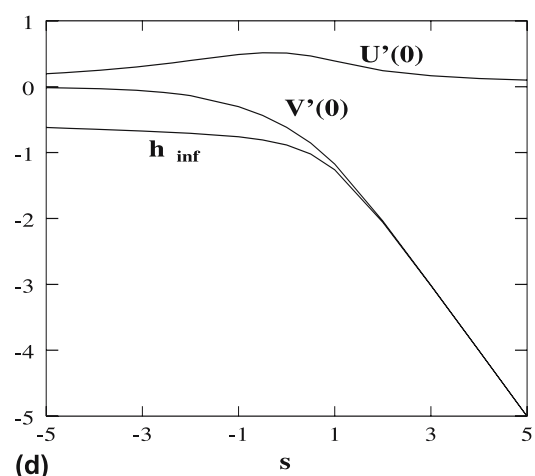

(d)

Figure 1. Basic flow quantities for the rotating-disk flow in consideration with particular suction and injection parameters are shown respectively in (a) the radial velocity profiles, (b) the circumferential velocity profiles, (c) the wall normal velocity profiles, (d) the wall derivatives $U^{\prime}(0), V^{\prime}(0)$, and the infinity value of axial velocity $h_{\text {inf }}$. Also, the crosses in (a) refer to the locations of inflexion point.
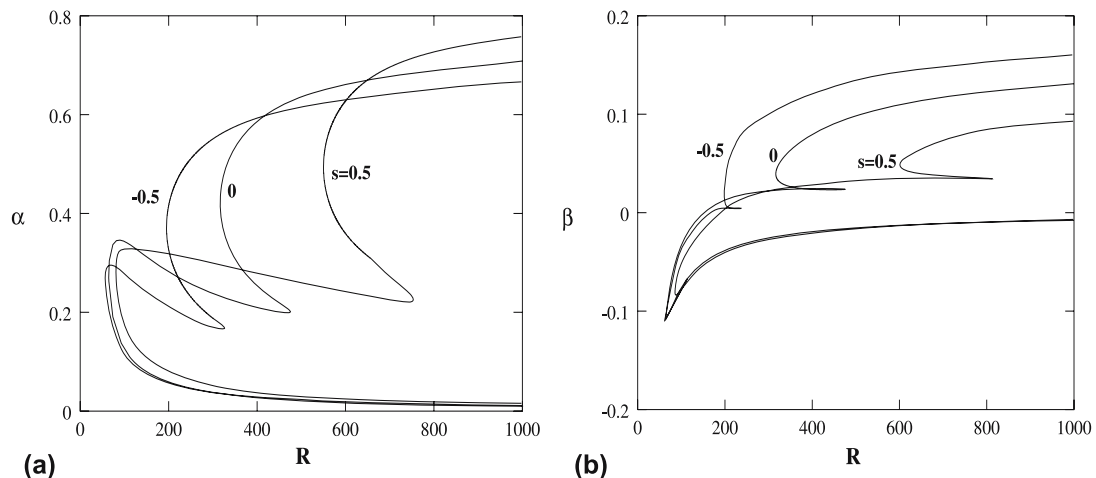

Figure 2. Neutral stability modes for the convective instability for travelling waves having the frequency $w=7.9$ are shown against the Reynolds number at three selected suction parameters, $s=-0 \cdot 5$, 0 and $0 \cdot 5$, respectively. (a) $\alpha$ versus $R$, (b) $\beta$ versus $R$. Convective instability starts to the right of the curves. 
Table 1. The critical values of Reynolds number as well as the related eigenvalues are tabulated for the onset of convective upper and lower branches (shown by the suffixes $u$ and $l$, respectively) as depicted in figure 2 at some selected suction/blowing parameters. The parameter $\varepsilon$ is defined as $\varepsilon=\arctan \left(\frac{\beta}{\alpha_{r}}\right)$.

\begin{tabular}{lcccccccc}
\hline$s$ & $R_{u}$ & $\alpha_{r u}$ & $\beta_{u}$ & $\varepsilon_{u}$ & $R_{l}$ & $\alpha_{r l}$ & $\beta_{l}$ & $\varepsilon_{l}$ \\
\hline-1 & 146.4 & 0.256 & 0.029 & 6.4 & 64.73 & 0.250 & -0.098 & -21.4 \\
-0.5 & 203.8 & 0.342 & 0.034 & $5 \cdot 7$ & 64.52 & 0.264 & -0.102 & -21.2 \\
0 & $316 \cdot 6$ & 0.421 & 0.039 & $5 \cdot 3$ & 64.44 & 0.276 & -0.106 & $-21 \cdot 1$ \\
0.5 & $635 \cdot 1$ & 0.445 & 0.04 & $5 \cdot 1$ & 82.3 & 0.265 & -0.078 & $-16 \cdot 3$ \\
1 & $1374 \cdot 2$ & 0.531 & 0.064 & 4.8 & 126.5 & 0.252 & -0.047 & -10.56 \\
\hline
\end{tabular}

one presented in Lingwood (1997) (see figure 4 of Lingwood (1997)). Such travelling waves were first observed experimentally by Federov et al (1976) and were later calculated theoretically by Balakumar \& Malik (1990) for the zero-suction case. In parallel to the findings of Lingwood (1997), suction significantly increases the critical Reynolds number for the convective instability of the upper branch modes, unlike the decreasing effect of injection. The critical Reynolds number for the lower branch mode is less sensitive to the changes in $s$. Table 1 presents the critical parameters for the upper and lower modes, respectively for a range of parameters, for $\omega=7.9$ waves. Next, in figure 3 the effects of mass transfer on the absolute growth of the inviscid absolute instability are displayed. There takes place a dramatic reduction in the case of suction regarding the size of the absolute instability region as well as the absolute growth, though the diminishing rate is slow as suction is further increased.

Stationary and non-stationary waves of upper and lower inviscid/viscous branches originate from the invisible roughness elements on the surface of the rigid disk and amplified by the free stream turbulence outside the boundary layer. The first triggering of the non-linearity and the subsequent transition to turbulence are generally realized as a result of the resonance of such stationary/travelling waves. For instance, the coalescence of the upper inviscid, lower viscous and a third mode corresponding to the inwardly propagating negative energy wave causes the absolute instability mechanism which was studied for the incompressible and compressible flows by Lingwood $(1995,1997)$ and Turkyilmazoglu et al (2000). In addition to this, the triad coupling of the three stationary/travelling waves was shown to exist by Bassom \& Hall (1991) for the inviscid incompressible Von Karman's flow. Moreover, the experimental works of Corke \& Knasiak (1996, 1998) clearly demonstrated that the transition might be due to the resonance of triad coupling between a non-stationary and two stationary waves. Furthermore, the coalescence of two modes originating initially at the same wavenumber plane from a spatio-temporal analysis causes direct spatial resonance with a second-order pole. As a result of the algebraic growth of such perturbations for small times or distances, whenever they are nearly neutral, transition is suspected to occur in Plane-Poiseulle and Blasius boundary layer flows, see for example Koch (1986) and Shanthini (1989). Such a modal coalescence was also shown to take place in the incompressible rotating-disk flow at a Reynolds number of 445 in Turkyilmazoglu \& Gajjar (2000) using parallel flow approximation. However, even if the non-parallelism was included in the simulation of the perturbations as in Davies \& Carpenter (2003), the global instability mechanism is suggested to occur via a convective type of instability as also caused by the direct spatial resonance, instead of the local absolute instability mechanism as suggested in Lingwood (1995). Thus we attempt here to extend the 


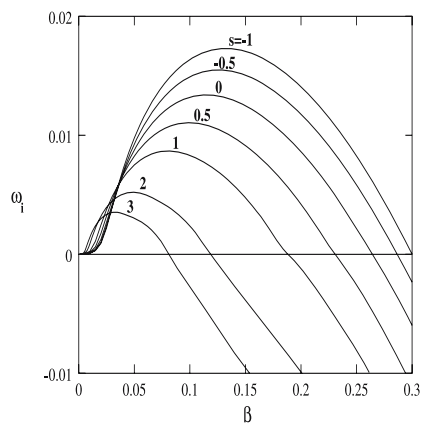

(a)

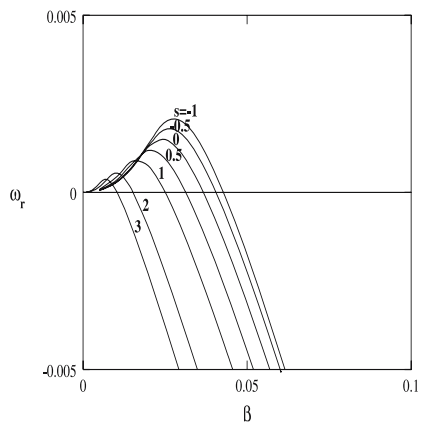

(b)

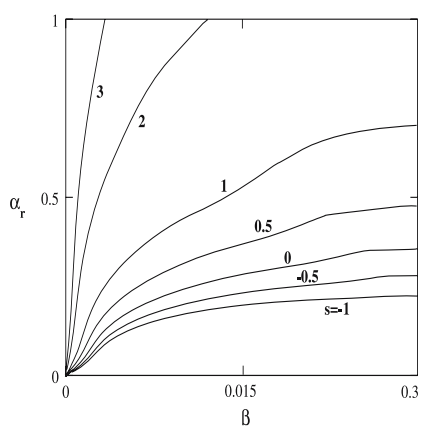

(c)

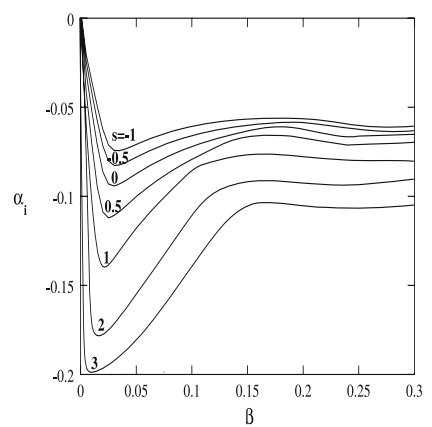

(d)

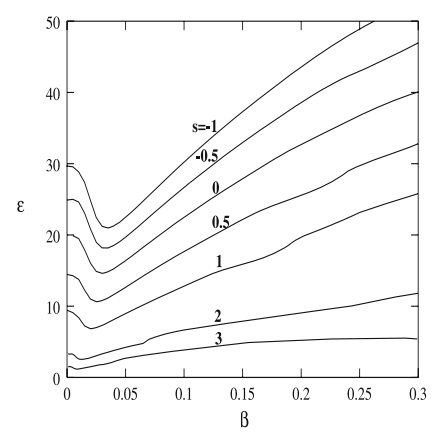

(e)

Figure 3. Marginal curves (satisfying the pinching requirement together with $\frac{\partial \omega}{\partial \alpha}=0$ ) for the occurrence of absolute instability are demonstrated at a variety of suction/injection parameters, obtained from the inviscid Rayleigh equation (3.2).

results of Turkyilmazoglu \& Gajjar (2000) to account for the effects of suction and injection on the direct spatial resonance instability mechanism.

In figure 4 a comparison of the marginal curves of direct spatial resonance for $s=-0.5$ and 0.5 with $s=0$ is made. When suction is applied with $s=0.5$ the critical Reynolds number for the onset of the resonance is increased by a factor of about 1.83 compared with the zerosuction case. Thus, suction has a significant stabilizing effect on the direct spatial resonance instability mechanism as for the absolute instability mechanism found in Lingwood (1997), though, it was more stabilizing in that case. Conversely, injection with $s=-0.5$ reduces the critical Reynolds number slightly making the flow more unstable as far as the direct spatial resonance instability is concerned. This outcome is strongly believed to be due to the position of the inflexion points of the mean velocity profiles as depicted in figure 1 . The criteria for the marginal curves as displayed in figure 4 are that the group velocity vanishes, i.e. $\frac{\partial \omega}{\partial \alpha}=0$ and $\omega_{i}=0$. Moreover, for an ordinary branch point to occur, in figure 5a the coalescence of two spatial branches (which are mainly the inviscid upper branch and the viscous lower branch) which originate initially in the same half- $\alpha$ plane are shown (see the fourth portion) for $s=0.5$. The critical branch point forms at the values $R=813.3, \beta=0.0396, \alpha_{r}=0.220$, $\omega_{r}=2.40$ and $\varepsilon=10 \cdot 2$, respectively. The first three portion of 5 correspond to a contour above all values of the temporal eigenvalues and the fourth one to a contour passing at the absolute frequency. The response of the basic flow to a localized line forcing at such a branch point will be measured by a Green's function whose asymptotic value after a usual 


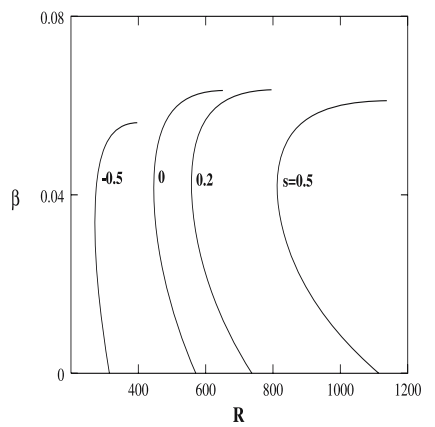

(a)

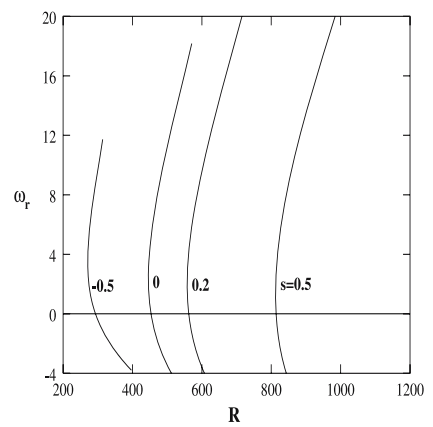

(b)

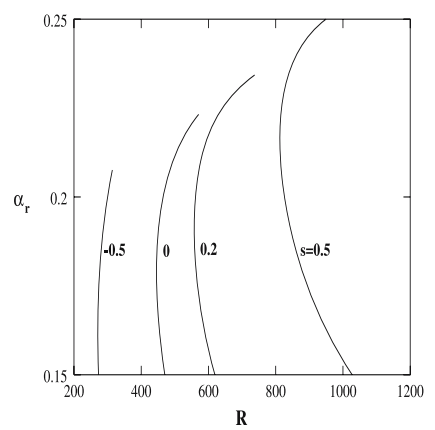

(c)

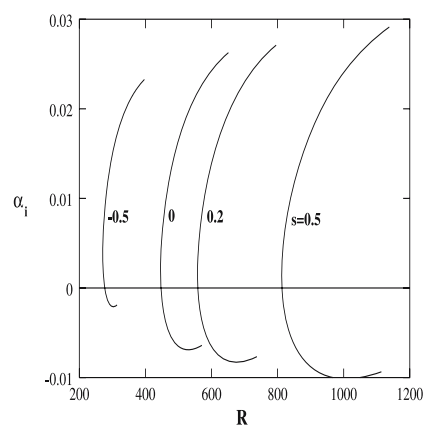

(d)

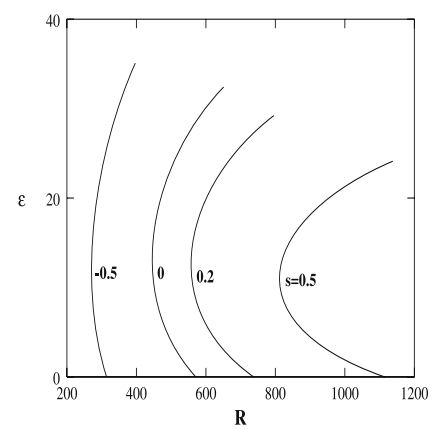

(e)

Figure 4. Marginal curves of ordinary branch points leading to direct spatial resonance instability for $\alpha_{i} \rightarrow 0$ in the viscous rotating-disk flow with some particular suction/injction parameters are demonstrated; (a) $\beta$, (b) $\omega_{r}$, (c) $\alpha_{r}$, (d) $\alpha_{i}$, (e) $\varepsilon$.

residue calculation demonstrates that the amplitude amplification of the disturbances at this resonance point can be evaluated as the inverse of $\alpha_{i}$ (refer to Carpenter \& Pedley (2003)), for which is practically zero. Thus, the algebraic growth of the transient disturbances within the presence of the direct spatial resonance might constitute a route to transition to turbulence in the rotating-disk boundary layer when mass transfer is in consideration.

Figure 6 summarizes the effects of suction/blowing on the onset of direct spatial resonance instability, giving only the critical values of the neutral values of marginal curves. We also present the critical values over the range $-1 \leq s \leq 2$ in table 2 for a selection of suction/ injection parameters. It should be remarked here that there occurs a second set of branch points leading to a resonance as also spotted by Lingwood (1997), but since they occur at larger Reynolds numbers wherein the flow is already in turbulent state, we find these irrelevant and hence omit these trajectories. Moreover, an integral equation for the three dimensional disturbances (6) to the undisturbed three dimensional velocity field (3) can be derived in order to establish some underlying physical mechanisms behind the effects of suction and blowing, leading to similar kinetic energy equations from the linearized momentum equations as also given in Cooper \& Carpenter (1997a) and Faller (1991). In accordance with these references, an analysis of the energy flux would indicate that the much larger stabilizing effect compared to the destabilizing effect of injection (see table 2) occurs through a reduction in energy production by the Reynolds shear stress and an increase in viscous dissipation.

Figure 7 shows $\alpha$ in the complex $\alpha$-plane at the critical Reynolds number for the onset of direct spatial resonance instability for a range of $s$. The solid lines in this figure indicate 


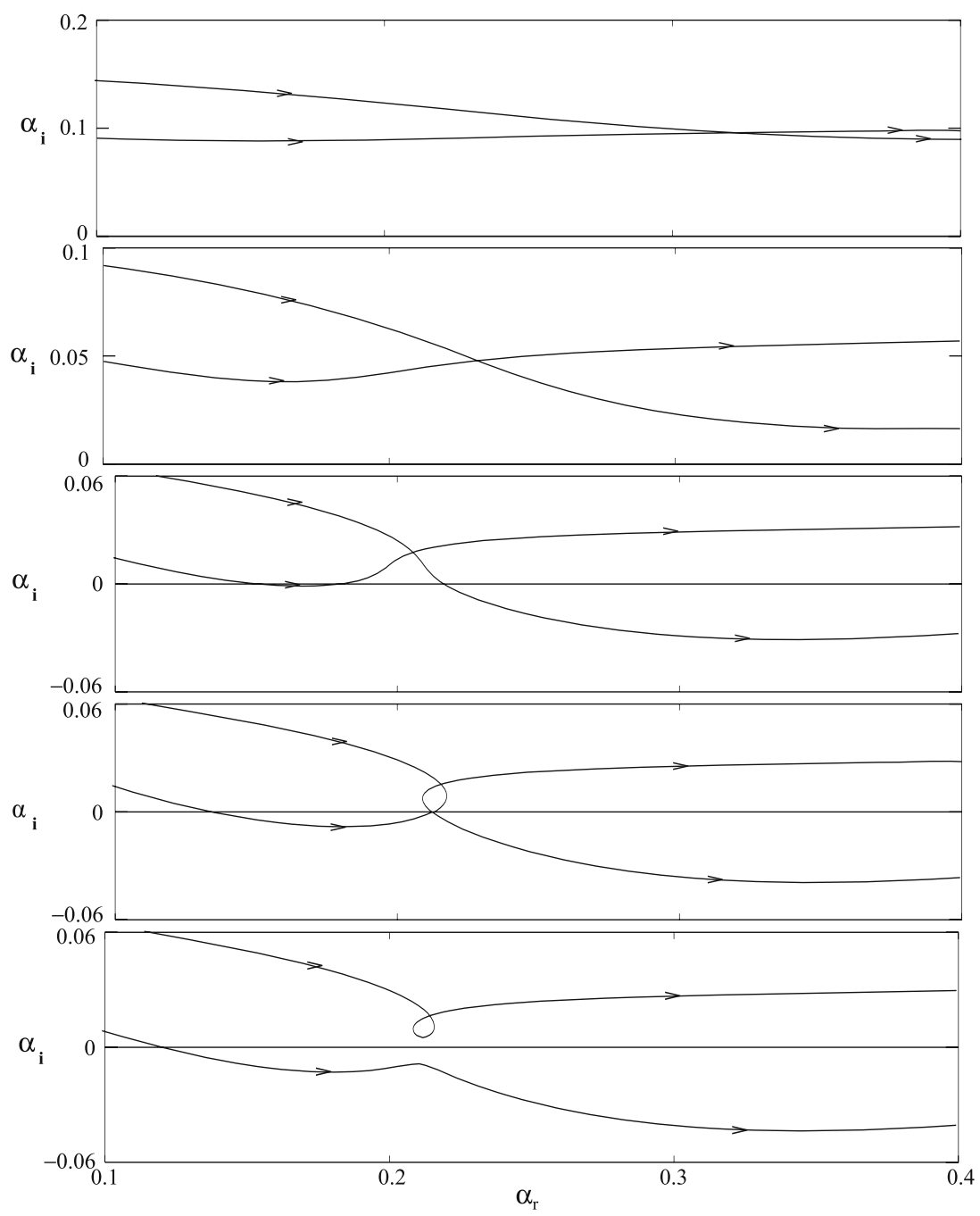

Figure 5. The progression of the two spatial branches at $R=813.3$ and $\beta=0.0396$ in the $\alpha$ plane is given in the viscous rotating-disk boundary-layer flow with suction $s=0 \cdot 5$. Graphs are for $\omega_{i}=6,3,0 \cdot 45,0$ and $-0 \cdot 02$, respectively. Branch point is at $\alpha=(0 \cdot 22,0) \omega=(2 \cdot 40,0)$ (fourth portion). A direct spatial resonance occurs between the two spatial branches since the corresponding $\alpha$ and $\omega$ are neutral. The direction of the arrows indicates the increasing frequency.

the emergence of the two spatial branches involved in each branch points as $\omega_{i} \rightarrow \infty$ away from the branch point. Since in each case the two spatial branches are born into the same half planes, as opposed to the separation into the distinct half planes for the absolute instability, these branch points constitute realistic direct spatial resonance instability.

Finally, in figure 8 we demonstrate the evolution of branch points as calculated from the inviscid Rayleigh solver. To make it clear that the high Reynolds number viscous spatial resonance modes match with inviscid Rayleigh modes, we track a viscous mode with increasing Reynolds number (taking $w_{i}=0$ ) in figure 9 for the case of impermeable wall and show that this mode asymptotically approaches the corresponding inviscid mode. It can be observed 

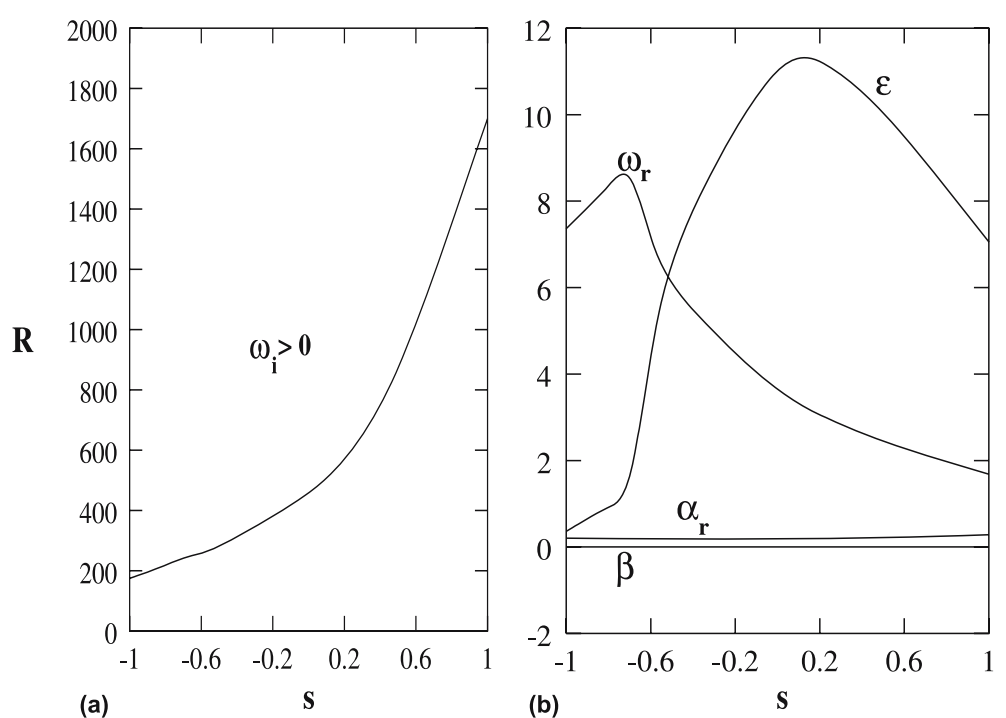

Figure 6. (a) Variation in the critical Reynolds number for the occurrence of direct spatial resonance instability with the mass transfer $s$ is shown. (b) Variation in the critical radial and azimuthal wavenumbers, wave angle, and frequency with $s$ is shown.

from the viscous modes that as Reynolds number tends to infinity, $\beta \rightarrow 0.07, \omega_{r} \rightarrow-0.006$, $\alpha_{r} \rightarrow-0.021$ and $\varepsilon \rightarrow-73 \cdot 3$. These values are seen to be well captured in figure 8 from the Rayleigh equation (see the parts with $\omega_{i}=0$ ). This, as well as the results as found in figure 3 in turn removes the possibility of being artifact for the modes obtained making use of the parallel-flow approximation.

The onset of absolute instability for zero suction is given by Lingwood (1995). It was found to occur at $R=507$ which is very close to the experimentally determined value of transition Reynolds number 513. Based on this, Lingwood (1996) suggested that the onset of absolute instability promotes the onset of non-linearity and laminar-turbulent transition. However, as argued in Turkyilmazoglu \& Gajjar (2000), the non-parallel effects are particularly significant near low Reynolds numbers with a destabilizing feature for growing boundary layers, see for example Smith (1979), Fasel \& Konzelmann (1990) and Malik (1994). Moreover,

Table 2. The critical values of Reynolds number as well as the related eigenvalues are tabulated for the onset of direct spatial resonance instability mechanism for $\alpha_{i} \rightarrow 0$ as depicted in figure 6 at some selected suction/blowing parameters.

\begin{tabular}{|c|c|c|c|c|c|c|}
\hline$s$ & $R_{c}$ & $\omega_{r c}$ & $\alpha_{r c}$ & $\alpha_{i c}$ & $\beta_{c}$ & $\varepsilon_{c}$ \\
\hline-1 & $174 \cdot 2$ & $7 \cdot 36$ & $0 \cdot 205$ & 0.007 & $0 \cdot 0013$ & $0 \cdot 36$ \\
\hline$-0 \cdot 5$ & $277 \cdot 1$ & $5 \cdot 86$ & $0 \cdot 180$ & $0 \cdot 0$ & 0.0225 & $7 \cdot 11$ \\
\hline 0 & $447 \cdot 2$ & $3 \cdot 54$ & $0 \cdot 185$ & $0 \cdot 0$ & 0.0376 & 11.49 \\
\hline 0.5 & $813 \cdot 3$ & 2.40 & 0.220 & $0 \cdot 0$ & 0.0396 & $10 \cdot 20$ \\
\hline 1 & 1700 & 1.68 & $0 \cdot 281$ & $0 \cdot 0$ & 0.0348 & 7.06 \\
\hline 2 & 3642 & 0.23 & 0.476 & 0.0 & 0.0252 & 3.03 \\
\hline
\end{tabular}




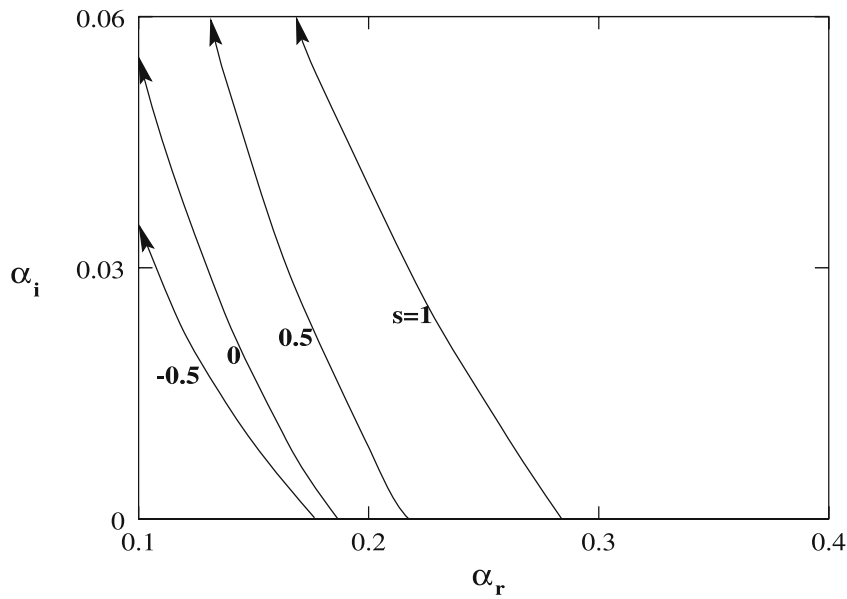

Figure 7. The development of the onset wavenumbers on two spatial branches that coalesce at each branch point as $\omega_{i} \rightarrow \infty$ (directions of the arrows) away from the values at the branch points is shown for some selected parameters $s$.

the recent work of Davies \& Carpenter (2003) indicates that the non-parallelism influences the absolute instability in a stabilizing manner. Additionally, as also stated in the work of Lingwood (1997), when the suction is considered, the onset of absolute instability is overestimated as compared to the onset of transition observed from the experiment of Gregory \& Walker (1960). This discrepancy was attributed to limitations in the experimental apparatus

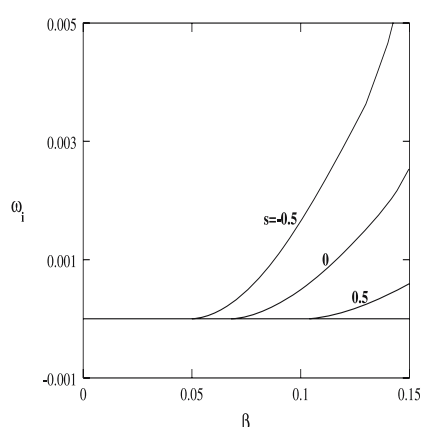

(a)

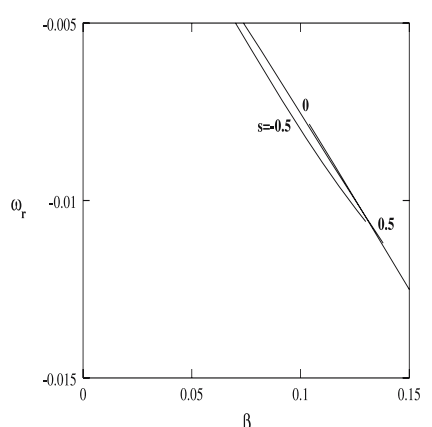

(b)

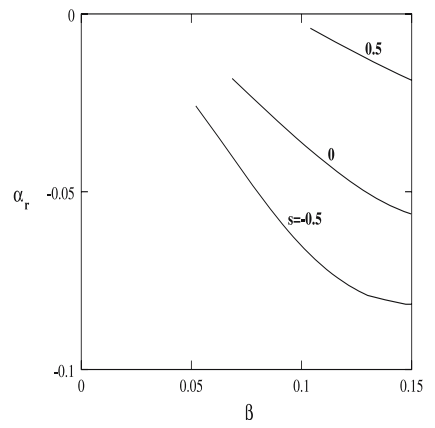

(c)

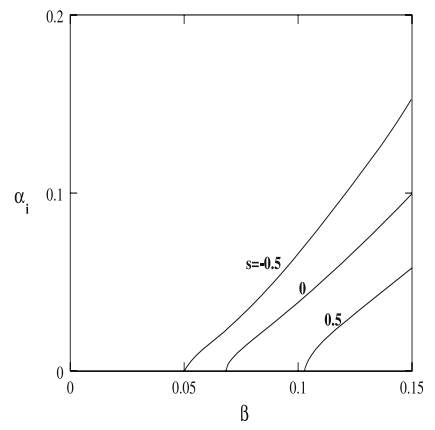

(d)

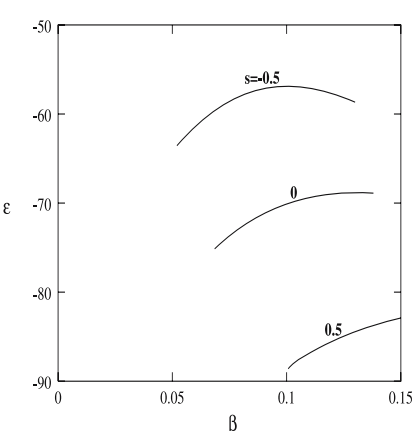

(e)

Figure 8. Marginal curves for the occurrence of direct spatial resonance instability are demonstrated at a variety of suction/injection parameters, obtained from the inviscid Rayleigh equation (3.2). 


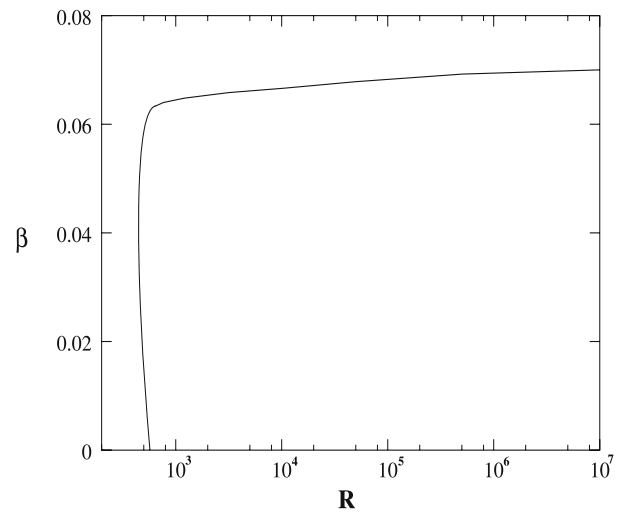

(a)

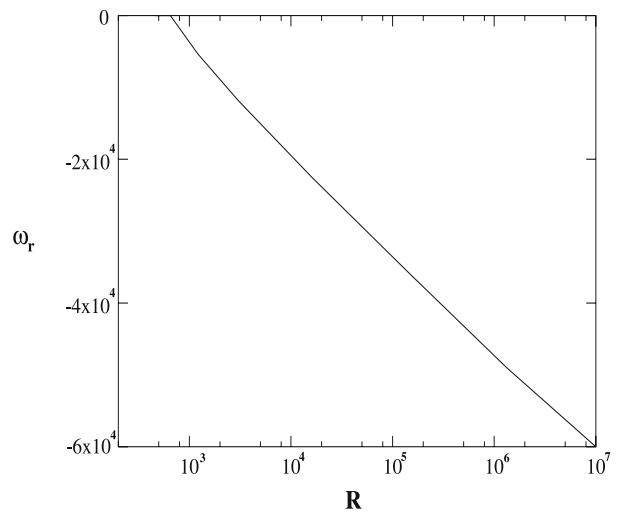

(b)

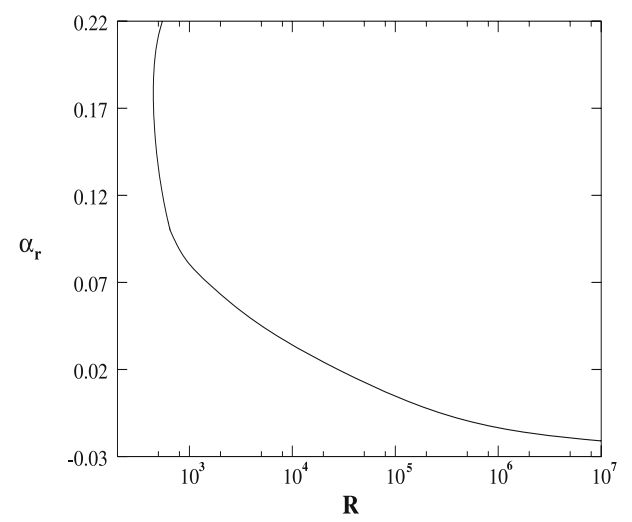

(c)

Figure 9. Marginal curves for the occurrence of direct spatial resonance instability are demonstrated for the impermeable wall case for large Reynolds numbers.

of Gregory \& Walker (1960), such as significant non-uniformity in suction, roughness of the disk and surface waviness reduced the effectiveness of the experimentally-achieved suction compared with that of uniform suction numerically determined. However, as found in the current study in the presence of suction the results of spatial resonance instability qualitatively support the outcomes of experiment of Gregory \& Walker (1960), see the comparison values as outlined in Lingwood (1997). Thus, we argue that in the absence of other mechanisms, the non-linearity will be promoted by the direct spatial resonance instability, which persists even the non-parallelism is included, and which will eventually drive the transition in the rotating-disk boundary layer flow.

\section{Conclusions}

In this paper an investigation has been performed into the theoretical behaviour of the laminar rotating-disk boundary layer flow within the consideration of mass transfer at the rigid disk using the linear stability theory. The effects of suction/injection on the convective instability as found earlier by Dhanak (1992), as well as on the inviscid absolute instability have been 
confirmed first. After that, the coalescing modes which originate in the same wavenumber plane and nearly neutral leading to direct spatial resonance instability have been searched. It has been found that this instability mechanism occurs on the rotating-disk boundary layer with uniform suction/injection applied on the wall. Moreover, it has been shown that suction has a stabilizing effect on this instability mechanism by increasing the critical Reynolds number, in contrast to a destabilizing effect of injection, as compared to the zero-suction case, an outcome dictated by the position of the inflexion point of the mean velocity profiles. Furthermore, the viscous resonance modes have been shown to match onto the inviscid ones removing the possibility that such a resonance might be an artifact of the parallel flow assumption during the linearization.

The recent investigation of Davies \& Carpenter (2003) clearly indicates that the local absolute instability mechanism is much affected by the action of non-parallelism. The works of Cooper \& Carpenter $(1997 \mathrm{a}, \mathrm{b})$ also points that under the effect of wall compliance, the complete suppression of the absolute instability mechanism is possible, but the effects on the spatial instability mechanisms seem poor. Thus, in the absence of the absolute instability mechanism the transition route to turbulence could be via the direct spatial resonance instability mechanism as determined here.

Meanwhile, it is known that the route to transition is via either the travelling waves which are excited by the free stream turbulence or the stationary waves which are excited by unavoidable roughnesses on the surface of the disk. In addition to this, it was shown by Bassom \& Seddougui (1992) that the rotating-disk boundary layer is too sensitive than the zero-suction case, because suction reduces the threshold amplitude for subcritical growth of such disturbances. Therefore, to highlight the importance of instability mechanisms, whether absolute or convective in the rotating-disk boundary layer with mass transfer, it would be necessary to perform particular experiments. It would further be useful to incorporate non-parallel and nonlinear influences, as done by Davies \& Carpenter (2003) for the zero-suction and Seddougui \& Bassom (1996) for mass transfer cases respectively, to assess the impacts of non-parallelism and non-linearity on the mass transfer through the disk.

\section{References}

Balachandar S, Street C L, Malik M R 1992 Secondary instability in rotating-disk flow. J. Fluid Mech. 242: 323-347

Balakumar P, Malik M R 1990 Travelling disturbances in rotating-disk flow. Theoret. Comput. Fluid Dyn. 2: 125-137

Bassom A P, Gajjar J S B 1988 Non-stationary crossflow vortices in a three dimensional boundary layer. Proc. Roy. Soc. London Ser. A417: 179-212

Bassom A P, Hall P 1991 Concerning the interaction of non-stationary crossflow vortices in a three dimensional boundary layer. Q. J. Mech. Appl. Math. 44: 147-172

Bassom A P, Seddougui S O 1992 The effects of suction on the non-linear stability of the threedimensional boundary layer above a rotating disc. Proc. Roy. Soc. London Ser. A436: 405

Benney D J, Gustavsson L H 1981 A new mechanism for linear and nonlinear hydrodynamic instability. Stud. Appl. Math. 64: 185-209

Bers A 1975 Linear waves and instabilities. Physique des Plasmas., 117-225

Briggs R J 1964 Electron-Stream Interaction With Plasmas (Cambridge, Mass: MIT Press)

Carpenter P W, Pedley T J 2003 Flow past highly compliant boundaries and in collapsible tubes. Kluwer Academic Publishers

Cooper A J, Carpenter P W 1997a The stability of rotating-disk boundary layer flow over a compliant wall. Part I. Type I and II instabilities. J. Fluid Mech. 350: 231-259 
Cooper A J, Carpenter P W 1997b The stability of rotating-disk boundary layer flow over a compliant wall. Part II. Absolute instability. J. Fluid Mech. 350: 261-270

Corke T C, Knasiak K F 1996 Crossflow instability with periodic distributed roughness. IUTAM Symposium on Laminar-Turbulent Transition 267-282

Corke T C, Knasiak K F 1998 Stationary travelling crossflow mode interactions on a rotating-disk. J. Fluid Mech. 355: 285-315

Davies C, Carpenter P W 2003 Global behaviour corresponding to the absolute instability of the rotating-disc boundary layer. J. Fluid Mech. 486: 287-329

Dhanak M R 1992 Effects of uniform suction on the stability of flow on a rotating disc. Proc. Roy. Soc. London Ser. A 439: 431

Faller A J 1991 Instability and transition of disturbed flow over a rotating-disk. J. Fluid Mech. 230: 245-269

Fasel H F, Konzelmann U 1990 Non-parallel stability of a fiat plate boundary layer using the complete Navier-Stokes equations. J. Fluid Mech. 221: 311-347

Federov B I, Plavnik G Z, Prokhorov I V, Zhukhovitskii L G 1976 Transitional flow conditions on a rotating-disk. J. Eng. Phys. 31: 1448-1453

Gregory N, Stuart J T, Walker W S 1955 On the stability of three dimensional boundary layers with applications to the flow due to a rotating-disk. Philos. Trans. R. Soc. London Ser. A 248: 155-199

Gregory N, Walker W S 1960 Experiments on the effects of suction the flow due to a rotating disk. J. Fluid Mech. 9: 225

Hall P 1986 An asymptotic investigation of the stationary modes of instability of the boundary layer on a rotating-disk. Proc. Roy. Soc. London Ser. A 406: 93-106

Hall P, Malik M R, Poll D I A 1984 On the stability of an infinite swept attachment-line boundary layer. Proc. Roy. Soc. London Ser. A 395: 229-245

Huerre P, Monkewitz P A 1990 Local and global instabilities in spatially developing flows. Ann. Rev. Fluid Mech. 22: 473-537

Kármán T V 1921 Uber laminare und turbulente reibung. Zeitschnnift fur angewantee Mathematik und Mechanik, 1: 233-252

Koch W 1986 Direct resonance in Orr-Sommerfeld equation. Acta Mech. 58: 11-29

Koch W 2002 On the spatio-temporal stability of primary and secondary crossflow vortices in a threedimensional boundary layer. J. Fluid Mech. 456: 85-111

Kohama Y, Ukaku M, Ohta F 1987 Boundary layer transition on a swept-cylinder. Proc. Int. Conf. Fluid Mech. 151-156

Lingwood R J 1995 Absolute instability of the boundary layer on a rotating-disk. J. Fluid Mech. 299: $17-33$

Lingwood R J 1996 An experimental study of absolute instability of the rotating-disk boundary layer flow. J. Fluid Mech. 314: 373-405

Lingwood R J 1997 On the effects of suction and injection on the absolute instability of the rotatingdisk boundary layer. Phys. Fluids. 9: 1317-1328

Mack L M 1984 Boundary layer linear stability theory. AGARD Rep. No. 709

Mack L M 1985 The wave pattern produced by a point source on a rotating-disk. AIAA Pap. No. 0490

Mackerrel S O 1987 A nonlinear asymptotic investigation of the stationary modes of instability of the 3 dimensional boundary layer on a rotating-disk. Proc. Roy. Soc. London Ser. A 413: 497-513

Malik M R 1986 The neutral curve for stationary disturbances in rotating-disk flow. J. Fluid Mech. 164: $275-287$

Malik M R, Li F, Chang C L 1994 Crossflow disturbances in three dimensional boundary layers: Nonlinear development, wave interactions and secondary instability. J. Fluid Mech. 268: 1-32

Malik M R, Poll D I A 1985 Effect of curvature on three dimensional boundary layer stability. AIAA J. 23: $1362-1369$

Malik M R, Zang T A, Hussaini M Y 1984 A spectral collocation method for the Navier-Stokes equations. J. Comput. Phys. 61: 64-88 
Seddougui S O 1990 A nonlinear investigation of the stationary mode of instability of the three dimensional compressible boundary layer due to rotating-disk. Q. J. Mech. Appl. Math. 43: 467-497

Seddougui S O, Bassom A P 1996 The effects of suction on the non-linear stability of a threedimensional compressible boundary layer. IMA J. Applied Math. 56: 183

Shanthini R 1989 Degeneracies of the temporal Orr-Sommerfeld eigenmodes in Plane-Poiseuille flow. J. Fluid Mech. 201: 13-34

Smith F T 1979 On the non-parallel flow stability of the Blasius boundary layer. Proc. Roy. Soc. London Ser. A 366: 91-109

Turkyilmazoglu M 1998 Linear absolute and convective instabilities of some two- and three dimensional flows. Ph.D. thesis, University of Manchester

Turkyilmazoglu M 2003 Instability of the flow in the vicinity of trailing edge of a class of thin aerofoils. Comput. Fluids 12: 353-371

Turkyilmazoglu M 2005 Effects of suction on the non-stationary lower branch modes of a compressible boundary layer flow. Stud. Appl. Math. 115: 357-385

Turkyilmazoglu M, Cole J W, Gajjar J S B 2000 Absolute and convective instabilities in the compressible boundary layer on a rotating disk. Theoret. Comput. Fluid Dyn. 14: 21-37

Turkyilmazoglu M, Gajjar J S B 2000 Direct spatial resonance in the laminar boundary layer due to a rotating-disk. Sādhanā-Acad. Proc. Eng. Sci. 25: 601-617

Wilkinson S P, Malik M R 1983 Stability experiments in rotating-disk flow. AIAA Pap. No. 1760

Wilkinson S P, Malik M R 1985 Stability experiments in the flow over a rotating-disk. AIAA J. 23: 588-595 